\title{
Dimensional reduction of dual topological theories
}

\author{
KASPER Olsen* \\ The Niels Bohr Institute \\ Blegdamsvej 17 \\ DK-2100 Copenhagen \\ Denmark
}

May 29, 2022

\begin{abstract}
We describe the reduction from four to two dimensions of the $S U(2)$ Donaldson-Witten theory and the dual twisted Seiberg-Witten theory, i.e. the Abelian topological field theory corresponding to the Seiberg-Witten monopole equations.
\end{abstract}

NBI-HE-96-14

hep-th/9603023

*E-mail: kolsen@nbivax.nbi.dk 
The recent discovery of duality in $N=2$ supersymmetric Yang-Mills theory [1] has a profound impact on the study of topological invariants of four-manifolds. The field theoretic tool, topological quantum field theory (for a nice review, see e.g. ref. [2]), was originally based on a non-Abelian gauge theory and associated ghost systems. This theory, from now on called Donaldson-Witten theory [3, 4], can be obtained from $N=2$ supersymmetric Yang-Mills theory by an appropriate twisting. If one instead twists the dual theory, weakly coupled in the infrared, one obtains the same information from a much simpler Abelian theory. In detail, one studies solutions to what has become known as the Seiberg-Witten monopole equations [5] (for an introduction, see e.g. ref. [6]). They involve an Abelian gauge potential $A_{\alpha}$ and a set of commuting Weyl spinors $M$ and $\bar{M}$. The explicit form of the associated topological Abelian field theory has been found [7].

Given these recent developments, a natural question to ask is what happens if one restricts the attention to four-dimensional product manifolds of the form $\mathcal{M}_{\triangle}=M_{n} \otimes S^{4-n}$ for $n=2,3$. Taking the radius of the circle $S$ to zero, one obtains dimensionally reduced theories which by construction are topological. Such dimensional reductions of Donaldson-Witten theory have been known for a long time [8, 9] (and is briefly reviewed below). As far as topological properties are concerned, the analogous dimensional reductions of the four-dimensional dual theory should provide new Abelian topological theories which are duals of the dimensionally reduced Donaldson-Witten theories.

The Donaldson-Witten theory in four dimensions is described by the following topological action, including a non-Abelian $S U(2)$ gauge potential $A_{\alpha}$ (see [四 for details):

$$
\begin{aligned}
S^{(4)}= & \int_{M} d^{4} x \sqrt{g} \operatorname{Tr}\left[\frac{1}{4} F_{\alpha \beta} F^{\alpha \beta}+\frac{1}{4} F_{\alpha \beta} \tilde{F}^{\alpha \beta}+\frac{1}{2} \phi D_{\alpha} D^{\alpha} \lambda-i \eta D_{\alpha} \psi^{\alpha}+2 i D_{\alpha} \psi_{\beta} \chi^{\alpha \beta}\right. \\
& \left.-\frac{i}{2} \lambda\left[\psi_{\alpha}, \psi^{\alpha}\right]-\frac{i}{2} \phi[\eta, \eta]-\frac{1}{8}[\phi, \lambda]^{2}\right]
\end{aligned}
$$

which is the BRST variation of

$$
V^{(4)}=\operatorname{Tr} F_{\alpha \beta}^{+} \chi^{\alpha \beta}-\operatorname{Tr} \frac{1}{2} B_{\alpha \beta} \chi^{\alpha \beta}+\frac{1}{2} \operatorname{Tr} \psi_{\alpha} D^{\alpha} \lambda-\frac{1}{4} \operatorname{Tr}(\eta[\phi, \lambda]) .
$$

$F_{\alpha \beta}^{+}$is the self-dual part of $F_{\alpha \beta}$, that is $F_{\alpha \beta}^{+}=\frac{1}{2}\left(F_{\alpha \beta}+\tilde{F}_{\alpha \beta}\right)$ with $\tilde{F}_{\alpha \beta}=\frac{1}{2} \epsilon_{\alpha \beta \gamma \delta} F^{\gamma \delta}$. The fields transform as

$$
\begin{array}{ll}
\delta A_{\alpha}=i \psi_{\alpha} & \\
\delta \psi_{\alpha}=-D_{\alpha} \phi & \\
\delta \phi=0 & \\
\delta \chi_{\alpha \beta}=B_{\alpha \beta} & \delta \lambda=2 i \eta \\
\delta B_{\alpha \beta}=0 & \delta \eta=\frac{1}{2}[\phi, \lambda]
\end{array}
$$

(the term $\phi[\chi, \chi]$ present in Wittens action [⿴囗十 can be included by adding to eq. (11) a $\delta$-exact term [10]). Note that $\delta^{2}=0$ only up to a gauge transformation, so $\delta^{2}=0$ on the gauge invariant action $S^{(4)}$. This is in fact enough to ensure that the theory is topological. The theory is defined on a four manifold $M$. Upon dimensionally reducing this action, we take $M$ to be a product manifold $M=Y \times S^{1}$ with signature $(++++)$ and assume that all fields are $x^{0}$-independent. Further we define $\chi^{i} \equiv \chi^{0 i}$ such that $\chi^{i j}=\epsilon^{i j k} \chi_{k}$. This leads to the three-dimensional action $(i, j, k=1,2,3)$,

$$
\begin{aligned}
S^{(3)}= & \int_{Y} d^{3} x \sqrt{g} \operatorname{Tr}\left[\frac{1}{4} F_{i j} F^{i j}+\frac{1}{2} F_{i j} \tilde{F}^{i j}+\frac{1}{2} D_{i} \varphi_{0} D^{i} \varphi_{0}-\frac{1}{2}\left[\varphi_{0}, \phi\right]\left[\varphi_{0}, \lambda\right]+\frac{1}{2} \phi D_{i} D^{i} \lambda\right. \\
& -i \eta D_{i} \psi^{i}-i \eta\left[\varphi_{0}, \psi_{0}\right]+2 i \epsilon^{i j k}\left(D_{i} \psi_{j}\right) \chi_{k}+2 i\left[\varphi_{0}, \psi_{i}\right] \chi^{i}+2 i \psi_{0} D_{i} \chi^{i} \\
& \left.-\frac{i}{2} \lambda\left[\psi_{0}, \psi_{0}\right]-\frac{i}{2} \lambda\left[\psi_{i}, \psi^{i}\right]-\frac{i}{2} \phi[\eta, \eta]-\frac{1}{8}[\phi, \lambda]^{2}\right] .
\end{aligned}
$$


where we defined $A_{0} \equiv \varphi_{0}$ and $\tilde{F}_{i j}=\epsilon_{i j k} F_{0 k}=-\epsilon_{i j k} D_{k} \varphi_{0}$. The reduction to two dimensions is obtained by assuming that the three manifold $Y$ is a product manifold of the form $Y=X \times S^{1}$ and $x^{1}$-independence of all fields $(\mu, \nu=2,3)$ :

$$
\begin{aligned}
S^{(2)}= & \int_{X} d^{2} x \sqrt{g} \operatorname{Tr}\left[\frac{1}{4} F_{\mu \nu} F^{\mu \nu}+\frac{1}{2} D_{\mu} \varphi_{0} D^{\mu} \varphi_{0}+\frac{1}{2} D_{\mu} \varphi_{1} D^{\mu} \varphi_{1}+\frac{1}{2}\left[\varphi_{1}, \varphi_{0}\right]^{2}\right. \\
& -\frac{1}{2}\left[\varphi_{0}, \phi\right]\left[\varphi_{0}, \lambda\right]-\frac{1}{2}\left[\varphi_{1}, \phi\right]\left[\varphi_{1}, \lambda\right]+\frac{1}{2} \phi D_{\mu} D^{\mu} \lambda-i \eta\left[\varphi_{0}, \psi_{0}\right]-i \eta\left[\varphi_{1}, \psi_{1}\right] \\
& -i \eta D_{\mu} \psi^{\mu}+2 i \epsilon^{\mu \nu}\left(D_{\mu} \psi_{\nu}\right) \chi+2 i \epsilon^{\mu \nu}\left[\varphi_{1}, \psi_{\mu}\right] \chi_{\nu}-2 i \epsilon^{\mu \nu}\left(D_{\mu} \psi_{1}\right) \chi_{\nu} \\
& +2 i\left[\varphi_{0}, \psi_{1}\right] \chi+2 i\left[\varphi_{0}, \psi_{\mu}\right] \chi^{\mu}+2 i \psi_{0} D_{\mu} \chi^{\mu}-2 i\left[\varphi_{1}, \psi_{0}\right] \chi \\
& -\frac{i}{2} \lambda\left[\psi_{0}, \psi_{0}\right]-\frac{i}{2} \lambda\left[\psi_{1}, \psi_{1}\right] \\
& -\frac{i}{2} \lambda\left[\psi_{\mu}, \psi^{\mu}\right]-\frac{i}{2} \phi[\eta, \eta]-\frac{1}{8}[\phi, \lambda]^{2} \\
& \left.+\frac{1}{2}\left[\varphi_{0}, \varphi_{1}\right] \epsilon^{\mu \nu} F_{\mu \nu}+\epsilon^{\mu \nu} D_{\mu} \varphi_{1} D_{\nu} \varphi_{0}\right]
\end{aligned}
$$

where we defined $A_{1} \equiv \varphi_{0}$ and $\chi_{1} \equiv \chi$. This action is also topological. In fact it is the variation of (traces in the adjoint representation implied)

$$
\begin{aligned}
V^{(2)}= & \left\{F_{\mu \nu} \epsilon_{\mu \nu}+2\left[\varphi_{0}, \varphi_{1}\right]\right\} \chi-2 B \chi+2\left\{\epsilon_{\mu \nu} D_{\nu} \varphi_{1}-D_{\mu} \varphi_{0}\right\} \chi_{\mu}-2 B_{\mu} \chi_{\mu} \\
& +\frac{1}{2} \psi_{\mu} D^{\mu} \lambda+\frac{1}{2} \psi_{0}\left[\varphi_{0}, \lambda\right]+\frac{1}{2} \psi_{1}\left[\varphi_{1}, \lambda\right]-\frac{1}{4}(\eta[\phi, \lambda])
\end{aligned}
$$

which is the dimensionally reduced version of the functional $V^{(4)}$ in eq. (2). In order to get a simple interpretation of the two-dimensional topological action, we define the complex scalar field $\Phi=\varphi_{0}+i \varphi_{1}$. It is then possible to rewrite the two-dimensional action as

$$
\begin{aligned}
S^{(2)}= & \int_{X} d^{2} x \sqrt{g} \operatorname{Tr}\left[\frac{1}{4}\left(F_{\mu \nu}-\frac{1}{2} i \epsilon_{\mu \nu}\left[\Phi, \Phi^{*}\right]\right)^{2}+\frac{1}{2} D_{\mu} \Phi D_{\mu} \Phi^{*}\right. \\
& -\frac{1}{2}\left[\varphi_{0}, \phi\right]\left[\varphi_{0}, \lambda\right]-\frac{1}{2}\left[\varphi_{1}, \phi\right]\left[\varphi_{1}, \lambda\right]+\frac{1}{2} \phi D_{\mu} D^{\mu} \lambda-i \eta\left[\varphi_{0}, \psi_{0}\right]-i \eta\left[\varphi_{1}, \psi_{1}\right] \\
& -i \eta D_{\mu} \psi^{\mu}+2 i \epsilon^{\mu \nu}\left(D_{\mu} \psi_{\nu}\right) \chi+2 i \epsilon^{\mu \nu}\left[\varphi_{1}, \psi_{\mu}\right] \chi_{\nu}-2 i \epsilon^{\mu \nu}\left(D_{\mu} \psi_{1}\right) \chi_{\nu} \\
& +2 i\left[\varphi_{0}, \psi_{1}\right] \chi+2 i\left[\varphi_{0}, \psi_{\mu}\right] \chi_{\mu}-2 i \psi_{0} D_{\mu} \chi^{\mu}-2 i\left[\varphi_{1}, \psi_{0}\right] \chi \\
& -\frac{i}{2} \lambda\left[\psi_{0}, \psi_{0}\right]-\frac{i}{2} \lambda\left[\psi_{1}, \psi_{1}\right] \\
& \left.-\frac{i}{2} \lambda\left[\psi_{\mu}, \psi_{\mu}\right]-\frac{i}{2} \phi[\eta, \eta]-\frac{1}{8}[\phi, \lambda]^{2}\right] .
\end{aligned}
$$

It is easy to check, that the resulting action is a BRST gauge fixing of the anti-self-duality equation in four dimensions, $F_{\alpha \beta}=-\frac{1}{2} \epsilon_{\alpha \beta \gamma \delta} F^{\gamma \delta}$, reduced to two dimensions. From the topological point of view, we could as well have gauge fixed on the self-duality equations (gauge fixing on $F^{-}$amounts essentially to extracting from eq. (1) the topological term $\frac{1}{2} \int F \tilde{F}$ and this changes neither the energy-momentum tensor nor the equations of motion). These equations have been studied by Hitchin in ref. [11] which focuses on the topological structure of the moduli space of solutions. Also Chapline and Grossman [9] considered these equations, thereby connecting conformal field theory to Donaldson theory.

We now turn to the main issue of this letter: the analogous dimensional reduction of the dual theory, which is an Abelian gauge theory. The fact, that it is Abelian implies enormous simplifications 
when it comes to actual calculations compared with its non-Abelian counterpart. The theory is defined by a topological action corresponding to the Seiberg-Witten equations in four dimensions [5]. These equations are

$$
\begin{aligned}
F_{\alpha \beta}^{+} & =-\frac{i}{2} \bar{M} \Gamma_{\alpha \beta} M \\
D_{A} M & =0
\end{aligned}
$$

Here $A$ is an Abelian $U(1)$ gauge potential, $D_{A}$ the twisted Dirac operator and $\Gamma_{\alpha \beta}=\frac{1}{2}\left[\gamma_{\alpha}, \gamma_{\beta}\right]$ with $\left\{\gamma_{\alpha}, \gamma_{\beta}\right\}=2 g_{\alpha \beta}$ I. $M$ is a (commuting) positive chirality spinor, satisfying $\gamma_{5} M=M$. As discussed in ref. [7, 12] one can write a completely analogous topological field theory based on these monopole equations (for a nice review, see e.g. ref. [13]). Let us use the notation of [12] where the topological action is

$$
S_{m}^{(4)}=\delta V_{m}^{(4)}
$$

with

$$
\begin{aligned}
V_{m}^{(4)}= & \int_{M} d^{4} x \sqrt{g}\left\{\left[\partial_{\alpha} \psi^{\alpha}+\frac{i}{2}(\bar{N} M-\bar{M} N)\right] \lambda-\chi^{\alpha \beta}\left(H_{\alpha \beta}-F_{\alpha \beta}^{+}-\frac{i}{2} \bar{M} \Gamma_{\alpha \beta} M\right)\right. \\
& \left.-\bar{\mu}\left(\nu-i D_{A} M\right)-\overline{\left(\nu-i D_{A} M\right)} \mu\right\} .
\end{aligned}
$$

The BRST transformation rules are,

$$
\begin{array}{ll}
\delta A_{\alpha}=\psi_{\alpha} & \\
\delta \psi_{\alpha}=-\partial_{\alpha} \phi & \delta M=N \\
\delta \phi=0 & \delta N=i \phi M \\
\delta \chi_{\alpha \beta}=B_{\alpha \beta} & \delta \mu=\nu \\
\delta B_{\alpha \beta}=0 & \delta \nu=i \phi \mu \\
\delta \lambda=\eta & \delta \eta=0
\end{array}
$$

As before $\delta^{2}=0$ up to a gauge transformation. For instance, $\delta^{2} A_{\alpha}=-\partial_{\alpha} \phi$ which is the variation of $A_{\alpha}$ under an infinitesimal gauge transformation generated by $\phi$. Ghost number assignments are essentially obvious, and can in any case be found in refs. [7, 12]. Using these transformation rules one finds the following expression for the topological action in four dimensions [12]:

$$
\begin{aligned}
S_{m}^{(4)}= & \int_{M} d^{4} x \sqrt{g}\left\{[-\Delta \phi+\bar{M} M \phi-i \bar{N} N] \lambda-\left[\partial_{\alpha} \psi^{\alpha}+\frac{i}{2}(\bar{N} M-\bar{M} N)\right] \eta+2 i \phi \bar{\mu} \mu\right. \\
& -\chi^{\alpha \beta}\left[\left(\partial_{\alpha} \psi_{\beta}-\partial_{\beta} \psi_{\alpha}\right)^{+}+\frac{i}{2}\left(\bar{M} \Gamma_{\alpha \beta} N+\bar{N} \Gamma_{\alpha \beta} M\right)\right] \\
& +\frac{1}{4}\left(F_{\alpha \beta}^{+}+\frac{i}{2} \bar{M} \Gamma_{\alpha \beta} M\right)^{2}+\frac{1}{2} \overline{D_{A} M} D_{A} M \\
& \left.+\overline{\left(i D_{A} N-\gamma^{\alpha} \psi_{\alpha} M\right)} \mu-\bar{\mu}\left(i D_{A} N-\gamma^{\alpha} \psi_{\alpha} M\right)\right\}
\end{aligned}
$$

where the Lagrange multipliers $B$ and $\nu$ are eliminated by their equations of motion and the bar indicates hermitian conjugation. By taking $M=Y \times S^{1}$ the dimensionally reduced action becomes:

\footnotetext{
${ }^{1}$ The convention is $D_{A}=\gamma^{\alpha}\left(\partial_{\alpha}+\omega_{\alpha}+i A_{\alpha}\right)$ so that $A_{\alpha}$ is real. $\omega_{\alpha}=\frac{1}{8} \omega_{\alpha i j}\left[\gamma^{i}, \gamma^{j}\right]$ is the spin connection one-form.
} 


$$
\begin{aligned}
S_{m}^{(3)}= & \int_{Y} d^{3} x \sqrt{g}\left\{[-\Delta \phi+\bar{M} M \phi-i \bar{N} N] \lambda-\left[\partial_{k} \psi^{k}+\frac{i}{2}(\bar{N} M-\bar{M} N)\right] \eta+2 i \phi \bar{\mu} \mu\right. \\
& -2 \chi^{k}\left[-\partial_{k} \psi_{0}+\epsilon_{k i j}\left(\partial_{i} \psi_{j}\right)-\bar{M} \sigma_{k} N-\bar{N} \sigma_{k} M\right] \\
& +\frac{1}{8}\left(F_{i j}-\epsilon_{i j k} \partial_{k} \varphi_{0}-\epsilon_{i j k} \bar{M} \sigma_{k} M\right)^{2}+\frac{1}{2} \overline{\left(D_{A}+\varphi_{0}\right) M}\left(D_{A}+\varphi_{0}\right) M \\
& \left.+\overline{\left(i\left(D_{A}+\varphi_{0}\right) N-\left(\sigma^{k} \psi_{k}-i \psi_{0}\right) M\right)} \mu-\bar{\mu}\left(i\left(D_{A}+\varphi_{0}\right) N-\left(\sigma^{k} \psi_{k}-i \psi_{0}\right) M\right)\right\}
\end{aligned}
$$

where $\chi^{i} \equiv \chi^{0 i}$ and $\sigma_{k}$ are the Pauli matrices. This dimensionally reduced action was obtained in ref. [12]. The partition function of this three dimensional theory gives a Seiberg-Witten version of the Casson invariant [12]. The monopole equations (8) reduce, as follows from eq. (13), to the following set of equations:

$$
\begin{aligned}
F_{i j}-\epsilon_{i j k} \bar{M} \sigma_{k} M & =0 \\
D_{A} M & =0 \\
\varphi_{0} & =0 .
\end{aligned}
$$

The last condition is only necessary if we have a non-trivial solution. If not, it can be replaced by the condition $d \varphi_{0}=0$. Finally, we make a reduction to two dimensions (with $Y=X \times S^{1}$ ) which results in the following action:

$$
\begin{aligned}
S_{m}^{(2)}= & \int_{X} d^{2} x \sqrt{g}\left\{[-\Delta \phi+\bar{M} M \phi-i \bar{N} N] \lambda-\left[\partial_{\mu} \psi^{\mu}+\frac{i}{2}(\bar{N} M-\bar{M} N)\right] \eta+2 i \phi \bar{\mu} \mu\right. \\
& -2 \chi^{\mu}\left[-\partial_{\mu} \psi_{0}+\epsilon_{\mu \nu} \partial_{\nu} \psi_{1}-\bar{M} \sigma_{\mu} N-\bar{N} \sigma_{\mu} M\right]-2 \chi\left[\epsilon_{\mu \nu}\left(\partial_{\mu} \psi_{\nu}\right)-\bar{M} \sigma_{1} N-\bar{N} \sigma_{1} M\right] \\
& +\frac{1}{8}\left(F_{\mu \nu}-\epsilon_{\mu \nu} \bar{M} \sigma_{1} M\right)^{2}+\frac{1}{4} \partial_{\mu} \varphi_{0} \partial_{\mu} \varphi_{0}+\frac{1}{4} \partial_{\mu} \varphi_{1} \partial_{\mu} \varphi_{1} \\
& +\frac{1}{2} \partial_{\mu} \varphi_{1} \epsilon_{\mu \nu} \bar{M} \sigma_{\nu} M+\frac{1}{2} \partial_{\mu} \varphi_{0} \bar{M} \sigma_{\mu} M+\frac{1}{4}\left(\bar{M} \sigma_{\mu} M\right)^{2} \\
& +\frac{1}{2} \overline{\left(D_{A}+\varphi_{0}+i \varphi_{1} \sigma_{1}\right) M}\left(D_{A}+\varphi_{0}+i \varphi_{1} \sigma_{1}\right) M \\
& +\overline{\left(i\left(D_{A}+\varphi_{0}+i \varphi_{1} \sigma_{1}\right) N-\left(\sigma^{\mu} \psi_{\mu}-i \psi_{0}+\psi_{1} \sigma_{1}\right) M\right)} \mu \\
& \left.-\bar{\mu}\left(i\left(D_{A}+\varphi_{0}+i \varphi_{1} \sigma_{1}\right) N-\left(\sigma^{\mu} \psi_{\mu}-i \psi_{0}+\psi_{1} \sigma_{1}\right) M\right)\right\}
\end{aligned}
$$

One can check that the resulting two-dimensional action also is topological since $S_{m}^{(2)}=\delta V_{m}^{(2)}$. Here $V_{m}^{(2)}$ is the dimensional reduction of $V_{m}^{(4)}$, i.e.

$$
\begin{aligned}
V_{m}^{(2)=} & \int_{X} d^{2} x \sqrt{g}\left\{\left[\partial_{\mu} \psi^{\mu}+\frac{i}{2}(\bar{N} M-\bar{M} N)\right] \lambda-2 \chi\left(2 H-\frac{1}{2} \epsilon_{\mu \nu} F_{\mu \nu}+\bar{M} \sigma_{1} M\right)\right. \\
& -2 \chi_{\mu}\left(2 H_{\mu}+\epsilon_{\mu \nu} \partial_{\nu} \varphi_{1}-\partial_{\mu} \varphi_{0}-\bar{M} \sigma_{\mu} M\right) \\
& \left.-\bar{\mu}\left(\nu-i\left(D_{A}+\varphi_{0}+i \varphi_{1} \sigma_{1}\right) M\right)-\overline{\left(\nu-i\left(D_{A}+\varphi_{0}+i \varphi_{1} \sigma_{1}\right) M\right)} \mu\right\} .
\end{aligned}
$$

Here $\chi \equiv \chi^{1}, H^{\mu} \equiv H^{0 \mu}$ and $H \equiv H^{1}$. The monopole equations are inferred from the classical part of the action. It is

$$
\begin{aligned}
S_{0}= & \frac{1}{8}\left(F_{\mu \nu}-\epsilon_{\mu \nu} \bar{M} \sigma_{1} M\right)^{2}+\frac{1}{4}\left(\partial_{\mu} \varphi_{1}\right)^{2}+\frac{1}{2}\left(\partial_{\mu} \varphi_{0}\right)^{2}+\frac{1}{4}\left(\bar{M} \sigma_{\mu} M\right)^{2} \\
& +\frac{1}{2}\left|D_{A} M\right|^{2}+\frac{1}{2}\left|\varphi_{0} M\right|^{2}+\frac{1}{2}\left|\varphi_{1} \sigma_{1} M\right|^{2}
\end{aligned}
$$


In two dimensions the monopole equations therefore reduce to

$$
\begin{aligned}
F_{\mu \nu}-\epsilon_{\mu \nu} \bar{M} \sigma_{1} M & =0 \\
D_{A} M & =0 \\
\bar{M} \sigma_{\mu} M & =0 \\
\varphi_{0} & =0 \\
\varphi_{1} & =0 .
\end{aligned}
$$

If $(A, M)$ is a trivial solution, then the last two conditions can be replaced by $d \varphi_{0}=d \varphi_{1}=0$. Due to the consistency of the monopole equations in four, three and two dimensions, the first of the above mentioned relations could as well have been deduced directly from the monopole equations (8) in four dimensions. For example, consider the $F_{01}^{+}$component of eq. (8). By dimensional reduction we get $\frac{1}{4} \epsilon_{\mu \nu} F^{\mu \nu}$ on the l.h.s and $\frac{1}{2} \bar{M} \sigma_{1} M$ on the r.h.s., which is the wanted relation. Similarly, the unusuallooking condition (18) is nothing but one of the dimensionally reduced remnants of the 3 -d monopole eqs. (14) and can be derived from the $F_{1 \mu}$ part of the first of these equations together with $\varphi_{1}=0$. It is possible to take $M$ to be a positive chirality spinor $\left(\sigma_{1} M=M\right)$, but this is not in it self dictated by the dimensional reduction.

Some explicit solutions to the monopole equations on $\mathbf{R}^{2}$ have been constructed in ref. [14] and the solutions turn out to be vortex configurations. They are singular, as are the analogous 3-d solutions given by Freund in ref. [15]. As noted by Witten in [5], the monopole equations admit no $L^{2}$ solutions on flat $\mathbf{R}^{n}, n \leq 4$.

The vanishing theorems of Witten [5] also have an analogous formulation in two dimensions, as noted by Thompson in ref. [16] where a brief discussion of the dimensional reduction of the monopole equations also can be found. In fact, it follows from eq. (18), that if $(A, M)$ is a solution of the two-dimensional monopole equations then the pair must obey the following identity

$$
\int_{X} d^{2} x \sqrt{g}\left(\frac{1}{4}|F|^{2}+\overline{D^{\mu} M} D_{\mu} M+\frac{1}{2}\left|\bar{M} \sigma_{1} M\right|^{2}+\frac{1}{4} R|M|^{2}\right)=0
$$

where $R$ is the scalar curvature. If there is a metric so that $R$ is positive on $X$ then this would imply that $F_{\mu \nu}=0$ and $M=0$ are the only solutions. On a sphere, for example, we are actually looking at flat Abelian connections.

In summary, we have found an expression for the topological action corresponding to the reduction of the monopole equations to two dimensions. As far as topological properties are concerned, this Abelian theory should be equivalent (dual) to the corresponding reduction of the Donaldson-Witten theory. Therefore one could speculate whether it is possible to extract from the Abelian theory information about the moduli space of solutions to the self-duality equations on a Riemann surface studied by Hitchin 11]. In connection with this, we may also refer to the work of Brooks and Lue 17, where it is shown that solutions to a twisted version of the four dimensional monopole equations are realized as a subset of solutions to the Donaldson-Witten theory. One may wonder whether a corresponding relation exists between the theory of Hitchin and the analogous twisting of the two dimensional monopole equations suggested here. It is intriguing to note that the two-dimensional topological theory corresponding to the monopole equations is very similar to the two-dimensional topological Abelian-Higgs model of Schaposnik and Thompson [18]. It is of interest to determine if they are in fact directly related. We hope to return to these questions in a future publication.

Acknowledgement: The author would like to thank J.M.F. Labastida for helpful discussions and remarks. 


\section{References}

[1] N. Seiberg and E. Witten, Nucl. Phys. B246 (1994) 19.

[2] D. Birmingham, M. Blau, M. Rakowski and G. Thompson, Phys. Rep. 209 (1991) 129.

[3] S. K. Donaldson, Topology 29 (1990) 257.

[4] E. Witten, Commun. Math. Phys. 117 (1988) 353.

[5] E. Witten, Math. Res. Lett. 1 (1994) 769.

[6] M. Marcolli, dg-ga/9509005.

[7] J.M.F. Labastida and M. Marino, Phys. Lett. B351 (1995) 146.

[8] D. Birmingham, M. Rakowski and G. Thompson, Nucl. Phys. B315 (1989) 577.

[9] G. Chapline and B. Grossman, Phys. Lett. B223 (1989) 336.

[10] L. Baulieu and I.M. Singer, Nucl. Phys. B (Proc. Suppl) 5B (1988) 12.

[11] N.J. Hitchin, Proc. London Math. Soc. 3 (1987) 59.

[12] A.L. Carey, J. McCarthy, B.L. Wang and R.B. Zhang, hep-th/9504005.

[13] J.M.F. Labastida, hep-th/9511037.

[14] S. Nergiz and C. Saçhog̃lu, hep-th/9602088.

[15] P.G.O. Freund, hep-th/9412208.

[16] G. Thompson, hep-th/9511038.

[17] R. Brooks and A. Lue, hep-th/9412206.

[18] A. Schaposnik and G. Thompson, Phys. Lett. B224 (1989) 379. 